UDC 577.125.54+615.272.4

DOI: 10.15587/2519-8025.2021.234699

\title{
OVERVIEW OF CONCEPTS OF THE SPHINGOLIPID METABOLISM
}

\author{
Galyna Storozhenko, Vitalina Kharchenko, Oksana Krasilnikova, Oksana Tkachenko
}

Sphingolipids are important components of the cell involved in the processes of apoptosis, inflammation, oncogenesis, aging, proliferation, differentiation and growth of cells, as well as in the stress-induced response of cells.

The aim. To study research literature for summarizing the new concepts of sphingolipids biochemical role in the development of various pathological conditions.

Materials and methods. The open sources of scientific literature were analyzed.

Results and discussion. According to the analyzed data, the occurrence of pathologies is associated with the sphingolipid imbalance in cells, and excessive accumulation of ceramides, while by preventing the accumulation of ceramides in cells, it is possible to prevent the appearance of cardiac, neurological and metabolic pathologies, including insulin resistance, heart disease (atherosclerosis, heart failure), as well as hepatic steatosis. Therefore, it is promising to search for drugs that can inhibit individual components of the metabolism of sphingolipids and prevent the development of pathology.

Conclusions. Sphingolipids are involved in numerous processes in cells, and changes in the balance of individual members of this class of lipids can play a crucial role in the development of pathological conditions. At the same time, the accumulated data on disorders of the sphingolipid metabolism in various diseases contribute to the development of drugs based on inhibition of the corresponding components of the metabolism of these lipids

Keywords: sphingolipids, ceramides, mitochondria, apoptosis, insulin resistance, viruses

How to cite:

Storozhenko, G., Kharchenko, V., Krasilnikova, O., Tkachenko, O. (2021). Overview of concepts of the sphingolipid metabolism. ScienceRise: Biological Science, 2 (27), 23-27. doi: http://doi.org/10.15587/2519-8025.2021.234699

(C) The Author(s) 2021

This is an open access article under the Creative Commons CC BY license

\section{Introduction}

Sphingolipids are important components of many biological processes in the cell. In recent years, the focus of research on these lipids has changed dramatically several times. Thus, for a long time sphingolipids were considered simply structural components of eukaryotic cell membranes. Then the results of research showed that some sphingolipids are crucial for many cellular processes as bioactive molecules and secondary messengers [1]. In addition, it was found that some representatives of this class of lipids have signalling properties, their role was determined in the stress-induced response of cells [2], apoptosis [3], processes of proliferation, differentiation and growth of cells, inflammation [4], oncogenesis [5, 6] and aging $[7,8]$. The critical role in the regulation of cellular processes through the signalling sphingomyelin pathway belongs to ceramide, which as a secondary messenger has antiproliferative properties, modulates the phosphorylation of various proteins and is an inducer of apoptosis [2]. It is known that the level of ceramide in cells increases significantly under conditions of natural aging. Thus, a number of studies have shown the accumulation of ceramide and an increase in the ratio of ceramides / sphingomyelin in the cells of an aging organism $[8,9]$. Recently, ceramides have been in the spotlight of scientists due to their role in various pathophysiological processes underlying cancer [10, 11], inflammation
$[4,12]$, depression [13] and neurodegenerative disorders [14], given Alzheimer's disease and dementia. In addition, later studies have highlighted their importance in obesity and diabetes $[15,16]$, as well as in cardiovascular disease [17]. It is suggested that the development of pathologies is associated with imbalance of sphingolipids in cells and excessive accumulation of ceramides, while preventing the accumulation of ceramides in cells may prevent the emergence of cardiac and metabolic pathologies, including insulin resistance, cardiovascular disease, cardiovascular disease, atherosclerosis hepatic steatosis $[18,19]$. At the same time, insufficient production of ceramide, known as Niemann-Pick syndrome, is caused by mutations in the acid lysosomal sphingomyelinase SMPD1 gene, which makes it impossible to convert sphingomyelin to ceramide [20]. It was also found that disorders of sphingolipid metabolism could directly or indirectly affect the functioning of mitochondria, provoking dysfunction of these organelles. In addition to the specific effects of ceramide outlined above, it is worth noting the central role of this lipid in the development of stress reactions in the cell. Ceramide is thought to coordinate the response to stress in a cell whose mechanism is universal for various eukaryotic organisms. However, sphingolipids perform various functions both at the level of the viral life cycle and in the regulation of antiviral immune responses [21, 22]. Such numerous and diverse 
effects of sphingolipids lead to increased interest of scientists in this group of lipids and enzymes involved in their metabolism, as potential targets for the development of new therapeutic agents.

The aim. To investigate scientific literature sources to formulate the latest views on the biochemical role of sphingolipids in the development of various pathological conditions.

\section{Materials and methods}

The study is devoted to the analysis of open sources of scientific literature using the scientific base PubMed.

\section{Research results branes \\ 3.1. Sphingolipids as components of cell mem-}

Sphingolipids are important components of cell membranes, and sphingomyelin is a structural lipid of the membrane bilayer, and its metabolites and precursors perform both structural and signalling functions [2, 23]. Ceramide is a metabolite of sphingomyelin and not only structures membrane clusters around the transmembrane protein, forming shelves, but also acts as a secondary mediator of signalling cascades, primarily related to the regulation of viability [2]. Ceramides are essential to ensure the structural integrity of plasma and mitochondrial membranes. Moving the ceramide molecule between the outer and inner surfaces of the membrane (flip-flop transitions) can change the configuration of the membranes and the affinity of the proteins to the membrane, which in turn affects the activity of membrane enzymes. It was found that a sharp increase in ceramide content in cells during the action of various toxic substances causes the activation of a number of signalling pathways (ERK, p38) involved in the implementation of apoptosis, and precedes the development of inflammatory reactions and cell death in various tissues [11]. Thus, mitogenactivated protein kinase p38 is involved in ceramideinduced cardiomyocyte apoptosis [24]. Biologically active sphingolipids can inhibit a number of molecules involved in the transmission of hormonal signals in cells such as Akt / protein kinase B and phospholipase D. In addition, ceramides synthesized in the endoplasmic reticulum can penetrate the mitochondria through membrane contacts and increase membrane permeability to cytochrome-c and adenylate kinase [25].

Other sphingolipids, such as sphingosine and sphinganine, also function as proapoptotic factors, so it is believed that the balance between ceramide, sphingosine1-phosphate and other proapoptotic sphingolipids is critical in the regulation of ceramide-induced programmed. Thus, sphingosine and ceramide induce cell cycle arrest and are involved in receptor-dependent apoptosis, whereas sphingosine-1-phosphate helps maintain cell viability during division by inhibiting differentiation [26].

In addition, different molecular species of ceramide differ in intracellular localization and biological effects [27]. Short-chain artificially synthesized ceramides (D-erythro-Nacetylsphingosine and N-hexanoyl-D-sphingosine) are thought to penetrate cells more easily and cause a greater toxic effect than long-chain natural analogues synthesized in the endoplasmic reticulum. These observations allow the use of short-chain ceramides in the treatment of some forms of cancer $[9,10]$.

\subsection{The effect of sphingolipids on mitochondria}

In most cell types, one of the key events in apoptosis is the release of proapoptotic proteins from the mitochondria into the cytoplasm. A detailed study of the mechanisms of apoptosis revealed that this process is facilitated by the formation of ceramide channels in the outer membrane of mitochondria [3]. It is known that ceramide, which is part of the plasma membrane, is important for its structural and functional organization. In addition to its structural function, ceramide can act as a secondary mediator in the transmission of various signals and has a high regulatory weight in apoptosis, inducing the release of proapoptotic proteins from mitochondria [3, 25].

Recently, many studies have emerged that suggest a strong relationship between ceramide metabolism, mitochondria, and separate mitochondria as a specialized compartment of sphingolipid metabolism with its own subpopulation of synthesizing and metabolizing ceramide enzymes. Thus, the detected content of various sphingolipids in mitochondria, taking into account sphingomyelin and ceramides [25], involves the creation of ceramide directly in these organelles. Subsequent studies of the submitochondrial activity of ceramide synthase have shown that ceramide can be synthesized on both the outer and inner membranes of mitochondria [28]. Recent studies characterize some isoforms of ceramides synthases (CerS1, CerS2, CerS4, and CerS6) in isolated mitochondria from mouse brains [29]. This also suggests that the synthesis of some ceramide-forming enzymes must be localized in the mitochondria. In addition, selective hydrolysis of the mitochondrial pool of sphingomyelin to form ceramide during the action of bacterial sphingomyelinase on the mitochondria causes apoptosis. Conversely, the generation of ceramides in the plasma membrane, endoplasmic reticulum, or Golgi apparatus during the action of sphingomyelinase on these cell compartments has no effect on cell viability. Undisputed evidence of the involvement of the mitochondrial pool of ceramide in apoptosis has been demonstrated in Caenorhabditis elegans nematode cells, where apoptosis induced by ionizing radiation was stopped by ceramide synthase inactivation and restored by microinjection of long-chain ceramides [30].

Indirect modulation of the functional state of mitochondria by ceramides may be mediated by a change in the ratio of pro- and antiapoptotic proteins of the $\mathrm{Bcl}-2$ family on the outer surface of the mitochondrial membrane. The direct effect of ceramide on mitochondria is possible due to the formation of channels for the release of cytochrome-c in the outer membrane of mitochondria, the opening of mitochondrial pores in the inner membrane of these organelles in the presence of $\mathrm{Ca}^{2+}$, as well as due to the activation of Bax on the outer membrane of mitochondria or suppression of the respiratory chain with the subsequent accumulation of reactive oxygen species. It has now been shown that ceramide can directly or indirectly affect the respiratory chain [23, 25, 29], causing severe suppression of the initial stage of the respiratory chain. Inhibition of the main flow of electrons in the respiratory chain stimulates the formation of reactive oxy- 
gen species, firstly, by increasing the lifetime of reduced electron transporters at the beginning of this chain and, secondly, by increasing intracellular oxygen concentration, which is no longer reduced by cytochrome oxidase. Stimulation of the formation of reactive oxygen species in the mitochondria causes the opening of the mitochondrial pore and the launch of a subsequent cascade of apoptosis.

\section{sistance}

\subsection{Sphingolipid metabolism and insulin re-}

The role of ceramides in the pathogenesis is determined not only by their participation in apoptotic signalling, but also in the work of other regulatory signalling cascades in the cell. It is known that ceramide can act on various components of the insulin signalling cascade and lead to impaired insulin function [31]. When synthetic analogues of ceramide (C2-ceramide, C6-ceramide) are added to cell culture, insulin-stimulated glucose uptake, translocation of glucose transporters, and / or glycogen synthesis are inhibited. In the culture of muscle cells, adipocytes and hepatocytes, synthetic $\mathrm{C} 2$ and C6-ceramides inhibit the activation of Akt / PKB, which underlies the rapid effects of glucose uptake and anabolic metabolism [32]. Ceramide suppresses this signalling step by two independent mechanisms. First, ceramide enhances dephosphorylation of Akt / PKB by direct activation of protein phosphatase 2A, which is responsible for dephosphorylation of protein kinase. Second, ceramide inhibits the translocation and activation of Akt / PKB by activating PKC $\zeta$, which phosphorylates Akt / PKB by an inhibitory residue contained in the $\mathrm{pH}$ domain of the enzyme [33]. In addition, ceramide inhibits phospholipase D, an integral part of insulinstimulated glucose metabolism. Thus, on human umbilical vein endothelial cells and human diploid fibroblasts, it was found that during the physiological aging of cells there is an increase in ceramide content, which is accompanied by impaired interaction of phospholipase D with PKC and / or ARF, as well as a significant decrease in phospholipase D activity [34].

Ceramide inhibits glycogen synthesis by inducing dephosphorylation and inhibition of Akt / PKB and preventing protein kinase translocation into the plasma membrane. Ceramide has been shown to enhance the phosphorylation of Thr-563/560-PKC $\xi / \lambda$, thereby mimicking the action of insulin and phosphatidylinositol3,4,5-triphosphate (a product of PI3 kinase) on aPKC in the liver of mice [35]. Moreover, C6-ceramide (but not its inactive analogue dihydro-C6-ceramide) induces PKC $\xi$ activity and also causes a selective enhancement of the association between Akt and PKC $\xi$ [36]. C6ceramide, like C2-ceramide, does not alter Akt phosphorylation in unstimulated cells and in cells, expressing dominant negative $\mathrm{PKC} \xi$, and reduces Akt phosphorylation in platelet-stimulated cell growth cells. These data suggest that ceramide-mediated excess activation of PKC $\xi$ leads to a decrease in Akt phosphorylation.

Thus, given the data of numerous studies, increasing the level of ceramides in morphologically and functionally different types of cells and tissues is a universal process in the development of metabolic syndrome, diabetes, cardiovascular and neurodegenerative diseases. Ceramides are involved in regulating the activity of key molecules involved in the insulin-signalling cascade. At the same time, the question remains as to the role of ceramide in the regulation of changes in insulin-dependent activation of phospholipase D in old age, both in classical targets of the hormone and in new ones, such as nervous tissue.

\subsection{Sphingolipids and viruses}

In addition to their important role in various processes in the cell, sphingolipids and their metabolites are potential key regulators of the life cycle of intracellular pathogens such as viruses. The role of sphingolipids and glycosphingolipids has not been considered for many years in the presence of viral infection due to difficulties in detecting or measuring these lipids. However, with the advent of lipid mass spectrometry, it has become possible to accurately determine the level of sphingolipids and glycosphingolipids in virus-infected cells and virus particles. Thus, Martin-Acebes and colleagues [37] proved that the particles of West Nile virus were enriched in sphingomyelin. At the same time, pharmacological inhibition of the activity of neutral sphingomyelinase (the enzyme that converts sphingomyelin to ceramide and phosphorylcholine) reduced the release of the virus from infected cells [37]. These data suggest that ceramide, which is formed due to catabolism of sphingomyelin, is critical for the life cycle of West Nile virus. A number of studies have indicated that viruses can use sphingolipids and / or glycosphingolipids to penetrate target cells, replicate their genome, or form new virus particles enriched in these lipids [21, 22, 38-40].

Sphingolipids can affect virus replication in three ways: 1 - acting as receptors during virus penetration; 2 - modulating virus replication and 3 - forming an antiviral immune response. Several studies have shown that sphingosine kinases and their sphingosine-1-phosphate product enhance the replication of influenza, measles, and hepatitis B virus. In contrast, ceramides, sphingosine 1-phosphate, and sphingosine kinase- 1 affect the expression of interferon and increasing the maturation, differentiation and location of dendritic cells in the tissue. It was also found that the synthetic molecule $\alpha$-galactosylceramide stimulates the activation of natural killer cells and the secretion of interferon- $\gamma$ [21, 22, 38]. Ceramide directly activates several enzymes and forms large, highly hydrophobic ceramide-enriched membrane domains that serve to reorganize receptors and signalling molecules $[39,40]$.

Thus, pharmacological inhibitors of the metabolic pathways of sphingolipids and glycosphingolipids have the potential to develop drugs with a broad spectrum of antiviral activity. Thus, it was found that pharmacological inhibition of acid sphingomyelinase by amitriptyline, imipramine, fluoxetine, sertraline, escitalopram, maprotiline or genetic reduction of the activity of this enzyme prevents infection of cultured cells or isolated human nasal epithelial cells with SARS-CoV-2 coronavirus and vesicular stomatitis virus [39]. In addition, the administration of anti-ceramide antibodies or neutral ceramidase also shows a significant protective effect in the case of SARS-CoV-2 infection. Anticeramide antibodies and neutral ceramidase are not currently approved for clinical use, but the authors believe that after clinical trials, drugs based on these inhibitors can be used as a nasal spray to prevent infections [39]. 


\section{Conclusions}

1. Sphingolipids, in particular ceramides, are involved in numerous cellular processes, and changes in the balance of individual members of this class of 1ipids can be crucial for the development of pathological conditions.

2. Many studies indicate a strong relationship between the metabolism of ceramide, sphingomyelin and mitochondria. It has been established that ceramide can modulate the functional state of mitochondria directly and indirectly. Thus, ceramide can directly or indirectly affect the respiratory chain, causing severe depression of the initial stage of the respiratory chain. In addition, the accumulation of ceramide in cells promotes the formation of channels in the mitochondrial membrane and the release of pro-apoptotic proteins to the cytoplasm.

3. Sphingolipids affect various components of the insulin-signalling cascade, so it was found that ceramide inhibits insulin-stimulated glucose uptake, translocation of glucose transporters and glycogen synthesis. Ceramides exert their effects on glucose metabolism by inhibiting the activation of Akt / PKB and phospholipase D in insulin target cells.

4. The content of ceramide in the cells of various tissues is significantly affected by the activity of enzymes of the sphingomyelinase group, and a number of studies indicate the leading role of the lysosomal pool of sphingomyelinases, the so-called acid sphingomyelinases. Thus, an increase in the activity of acid sphingomyelinases in various tissues of the aging body and in the presence of pathologies. At the same time, inhibition of the activity of acid sphingomyelinases by their excessive activation can have a number of therapeutic effects in the treatment of some pathological conditions and prevent virus infection.

\section{Conflict of interests}

The authors declare that they have no conflicts of interest.

\section{Financing}

The study was performed without financial support.

\section{References}

1. McQuiston, T., Haller, C., Poeta, M. (2006). Sphingolipids as Targets for Microbial Infections. Mini-Reviews in Medicinal Chemistry, 6 (6), 671-680. doi: http://doi.org/10.2174/138955706777435634

2. Hannun, Y. A., Obeid, L. M. (2008). Principles of bioactive lipid signalling: lessons from sphingolipids. Nature Reviews Molecular Cell Biology, 9 (2), 139-150. doi: http://doi.org/10.1038/nrm2329

3. Patwardhan, G. A., Beverly, L. J., Siskind, L. J. (2015). Sphingolipids and mitochondrial apoptosis. Journal of Bioenergetics and Biomembranes, 48(2), 153-168. doi: http://doi.org/10.1007/s10863-015-9602-3

4. Gomez-Muñoz, A., Presa, N., Gomez-Larrauri, A., Rivera, I.-G., Trueba, M., Ordoñez, M. (2016). Control of inflammatory responses by ceramide, sphingosine 1-phosphate and ceramide 1-phosphate. Progress in Lipid Research, 61, 51-62. doi: http://doi.org/10.1016/ j.plipres.2015.09.002

5. Zhu, S., Xu, Y., Wang, L., Liao, S., Wang, Y., Shi, M. et. al. (2021). Ceramide kinase mediates intrinsic resistance and inferior response to chemotherapy in triple- negative breast cancer by upregulating Ras/ERK and PI3K/Akt pathways. Cancer Cell International, 21 (1). doi: http://doi.org/10.1186/s12935-020-01735-5

6. Nganga, R., Oleinik, N., Ogretmen, B. (2018). Mechanisms of Ceramide-Dependent Cancer Cell Death. Sphingolipids in Cancer, 1-25. doi: http://doi.org/10.1016/bs.acr.2018.04.007

7. Babenko, N. A., Garkavenko, V. V., Storozhenko, G. V., Timofiychuk, O. A. (2016). Role of acid sphingomyelinase in the age-dependent dysregulation of sphingolipids turnover in the tissues of rats. General Physiology and Biophysics, 35 (2), $195-205$. doi: http://doi.org/10.4149/gpb_2015046

8. Babenko, N. A., Kharchenko, V. S. (2013). Age-Related Changes in the Phospholipase D-Dependent Signal Pathway of Insulin in the Rat Neocortex. Neurophysiology, 45 (2), 120-127. doi: http://doi.org/10.1007/s11062-013-9346-9

9. Babenko, N. A., Storozhenko, G. V. (2017). Role of ceramide in the aging-related decrease of cardiolipin content in the rat heart. Advances in Gerontology, 7 (3), 195-200. doi: http://doi.org/10.1134/s207905701703002x

10. Grbčić, P., Car, E. P. M., Sedić, M. (2020). Targeting Ceramide Metabolism in Hepatocellular Carcinoma: New Points for Therapeutic Intervention. Current Medicinal Chemistry, 27 (39), 6611-6627. doi: http://doi.org/ 10.2174/0929867326666190911115722

11. Kartal Yandım, M., Apohan, E., Baran, Y. (2012). Therapeutic potential of targeting ceramide/glucosylceramide pathway in cancer. Cancer Chemotherapy and Pharmacology, 71 (1), 13-20. doi: http://doi.org/10.1007/s00280-012-1984-x

12. Edsfeldt, A., Dunér, P., Ståhlman, M., Mollet, I. G., Asciutto, G., Grufman, H. et. al. (2016). Sphingolipids Contribute to Human Atherosclerotic Plaque Inflammation. Arteriosclerosis, Thrombosis, and Vascular Biology, 36(6), 1132-1140. doi: http://doi.org/10.1161/ atvbaha.116.305675

13. Dinoff, A., Herrmann, N., Lanctôt, K. L. (2017). Ceramides and depression: A systematic review. Journal of Affective Disorders, 213, 35-43. doi: http://doi.org/10.1016/j.jad.2017.02.008

14. Wang, G., Bieberich, E. (2018). Sphingolipids in neurodegeneration (with focus on ceramide and S1P). Advances in Biological Regulation, 70, 51-64. doi: http://doi.org/10.1016/j.jbior.2018.09.013

15. Field, B. C., Gordillo, R., Scherer, P. E. (2020). The Role of Ceramides in Diabetes and Cardiovascular Disease Regulation of Ceramides by Adipokines. Frontiers in Endocrinology, 11. doi: http://doi.org/10.3389/fendo.2020.569250

16. Fang, Z., Pyne, S., Pyne, N. J. (2019). Ceramide and sphingosine 1-phosphate in adipose dysfunction. Progress in Lipid Research, 74, 145-159. doi: http://doi.org/10.1016/j.plipres.2019.04.001

17. Li, N., Zhang, F. (2016). Implication of sphingosin-1-phosphate in cardiovascular regulation. Frontiers in Bioscience, 21 (7), 1296-1313. doi: http://doi.org/10.2741/4458

18. Pralhada Rao, R., Vaidyanathan, N., Rengasamy, M., Mammen Oommen, A., Somaiya, N., Jagannath, M. R. (2013). Sphingolipid Metabolic Pathway: An Overview of Major Roles Played in Human Diseases. Journal of Lipids, 2013, 1-12. doi: http://doi.org/10.1155/2013/178910

19. Apostolopoulou, M., Gordillo, R., Koliaki, C., Gancheva, S., Jelenik, T., De Filippo, E. et. al. (2018). Specific Hepatic Sphingolipids Relate to Insulin Resistance, Oxidative Stress, and Inflammation in Nonalcoholic Steatohepatitis. Diabetes Care, 41 (6), 1235-1243. doi: http://doi.org/10.2337/dc17-1318

20. Bajwa, H., Azhar, W. (2021). Niemann-Pick Disease. StatPearls. Treasure Island (FL): StatPearls Publishing. 
21. Schneider-Schaulies, J., Schneider-Schaulies, S. (2013). Viral Infections and Sphingolipids. Handbook of Experimental Pharmacology. Vienna: Springer, 321-340. doi: http://doi.org/10.1007/978-3-7091-1511-4_16

22. Bezgovsek, J., Gulbins, E., Friedrich, S.-K., Lang, K. S., Duhan, V. (2018). Sphingolipids in early viral replication and innate immune activation. Biological Chemistry, 399 (10), 1115-1123. doi: http://doi.org/10.1515/hsz-2018-0181

23. Hernández-Corbacho, M. J., Salama, M. F., Canals, D., Senkal, C. E., Obeid, L. M. (2017). Sphingolipids in mitochondria. Biochimica et Biophysica Acta (BBA) - Molecular and Cell Biology of Lipids, 1862 (1), 56-68. doi: http://doi.org/10.1016/j.bbalip.2016.09.019

24. Kong, J. Y., Klassen, S. S., Rabkin, S. W. (2005). Ceramide activates a mitochondrial p38 mitogen-activated protein kinase: A potential mechanism for loss of mitochondrial transmembrane potential and apoptosis. Molecular and Cellular Biochemistry, 278 (1-2), 39-51. doi: http://doi.org/10.1007/s11010-005-1979-6

25. Novgorodov, S. A., Wu, B. X., Gudz, T. I., Bielawski, J., Ovchinnikova, T. V., Hannun, Y. A., Obeid, L. M. (2011). Novel Pathway of Ceramide Production in Mitochondria. Journal of Biological Chemistry, 286 (28), 25352-25362. doi: http://doi.org/10.1074/ jbc.m110.214866

26. Dyatlovitskaya, E. V. (2007). The role of lysosphingolipids in the regulation of biological processes. Biochemistry (Moscow), 72 (5), 479-484. doi: http://doi.org/10.1134/s0006297907050033

27. Hannun, Y. A., Obeid, L. M. (2011). Many Ceramides. Journal of Biological Chemistry, 286 (32), 27855-27862. doi: http://doi.org/10.1074/jbc.r111.254359

28. Bionda, C., Portoukalian, J., Schmitt, D., Rodriguez-Lafrasse, C., Ardail, D. (2004). Subcellular compartmentalization of ceramide metabolism: MAM (mitochondria-associated membrane) and/or mitochondria? Biochemical Journal, 382 (2), $527-533$. doi: http://doi.org/10.1042/bj20031819

29. Yu, J., Novgorodov, S. A., Chudakova, D., Zhu, H., Bielawska, A., Bielawski, J. et. al. (2007). JNK3 Signaling Pathway Activates Ceramide Synthase Leading to Mitochondrial Dysfunction. Journal of Biological Chemistry, 282 (35), 25940-25949. doi: http://doi.org/10.1074/jbc.m701812200

30. Deng, X., Yin, X., Allan, R., Lu, D. D., Maurer, C. W., Haimovitz-Friedman, A. et. al. (2008). Ceramide Biogenesis Is Required for Radiation-Induced Apoptosis in the Germ Line of C. elegans. Science, 322 (5898), 110-115. doi: http://doi.org/10.1126/science.1158111

31. Yang, G., Badeanlou, L., Bielawski, J., Roberts, A. J., Hannun, Y. A., Samad, F. (2009). Central role of ceramide biosynthesis in body weight regulation, energy metabolism, and the metabolic syndrome. American Journal of Physiology-Endocrinology and Metabolism, 297 (1), E211-E224. doi: http://doi.org/10.1152/ajpendo.91014.2008

32. Park, M., Kaddai, V., Ching, J., Fridianto, K. T., Sieli, R. J., Sugii, S., Summers, S. A. (2016). A Role for Ceramides, but Not Sphingomyelins, as Antagonists of Insulin Signaling and Mitochondrial Metabolism in C2C12 Myotubes. Journal of Biological Chemistry, 291 (46), 23978-23988. doi: http://doi.org/10.1074/jbc.m116.737684

33. Powell, D. J., Turban, S., Gray, A., Hajduch, E., Hundal, H. S. (2004). Intracellular ceramide synthesis and protein kinase C $\zeta$ activation play an essential role in palmitate-induced insulin resistance in rat L6 skeletal muscle cells. Biochemical Journal, 382 (2), 619-629. doi: http://doi.org/10.1042/bj20040139

34. Webb, L. M., Arnholt, A. T., Venable, M. E. (2009). Phospholipase D modulation by ceramide in senescence. Molecular and Cellular Biochemistry, 337 (1-2), 153-158. doi: http://doi.org/10.1007/s11010-009-0294-z

35. Chen, F., Ghosh, A., Shneider, B. L. (2013). Phospholipase D2 mediates signaling by ATPase class I type 8B membrane 1. Journal of Lipid Research, 54 (2), 379-385. doi: http://doi.org/10.1194/jlr.m030304

36. Ivey, R. A., Sajan, M. P., Farese, R. V. (2014). Requirements for Pseudosubstrate Arginine Residues during Autoinhibition and Phosphatidylinositol 3,4,5-(PO4)3-dependent Activation of Atypical PKC. Journal of Biological Chemistry, 289 (36), 25021-25030. doi: http://doi.org/10.1074/jbc.m114.565671

37. Martin-Acebes, M. A., Merino-Ramos, T., Blazquez, A.-B., Casas, J., Escribano-Romero, E., Sobrino, F., Saiz, J.-C. (2014). The Composition of West Nile Virus Lipid Envelope Unveils a Role of Sphingolipid Metabolism in Flavivirus Biogenesis. Journal of Virology, 88 (20), 12041-12054. doi: http://doi.org/10.1128/jvi.02061-14

38. Yager, E. J., Konan, K. V. (2019). Sphingolipids as Potential Therapeutic Targets against Enveloped Human RNA Viruses. Viruses, 11 (10), 912. doi: http://doi.org/10.3390/v11100912

39. Carpinteiro, A., Edwards, M. J., Hoffmann, M., Kochs, G., Gripp, B., Weigang, S. et. al. (2020). Pharmacological Inhibition of Acid Sphingomyelinase Prevents Uptake of SARS-CoV-2 by Epithelial Cells. Cell Reports Medicine, 1 (8), 100142. doi: http://doi.org/10.1016/j.xcrm.2020.100142

40. Simonis, A., Schubert-Unkmeir, A. (2018). The role of acid sphingomyelinase and modulation of sphingolipid metabolism in bacterial infection. Biological Chemistry, 399 (10), 1135-1146. doi: http://doi.org/10.1515/hsz-2018-0200

Received date 06.05.2021

Accepted date 08.06.2021

Published date 30.06.2021

Galyna Storozhenko*, PhD, Assistant, Department of Biological Chemistry, National University of Pharmacy, Pushkinska str., 53, Kharkiv, Ukraine, 61002

Vitalina Kharchenko, PhD, Associate Professor, Department of Biochemistry, V. N. Karazin Kharkiv National University, Svobody sq., 4, Kharkiv, Ukraine, 61022

Oksana Krasilnikova, $\mathrm{PhD}$, Associate Professor, Department of Biological Chemistry, National University of Pharmacy, Pushkinska str., 53, Kharkiv, Ukraine, 61002

Oksana Tkachenko, PhD, Assistant, Department of Biological Chemistry, National University of Pharmacy, Pushkinska str., 53, Kharkiv, Ukraine, 61002

*Corresponding author: Galyna Storozhenko, e-mail: galyna.storozhenko@gmail.com 